\title{
THE BAER SUM FUNCTOR AND ALGEBRAIC $K$-THEORY
}

\author{
BY
}

IRWIN S. PRESSMAN( $\left.{ }^{1}\right)$

\begin{abstract}
The Baer sum operation can be described in such a way that it becomes a functorial product on categories of exact sequences of a fixed length. This product is proven to be coherently associative and commutative. The Grothendieck groups and Whitehead groups of some of these categories are computed.
\end{abstract}

1. Introduction. In the definitions of the Grothendieck and Whitehead groups of an abstract category, with respect to some fixed product on the category, it was necessary to assume that this product be a functor which is coherently associative and commutative [1], [5]. However, these products are generally taken to be tensor products or coproducts. We shall describe a functorialization of Reinhold Baer's classical sum operation on two extensions of the same groups, as another product which is useful to algebraic $K$-theory. This Baer sum functor will be called the Baer functor and will be denoted by $\boldsymbol{B}$.

$\boldsymbol{B}$ is defined and its properties are carefully described in $\$ \S 2$ and 3. Computations are done in $\S \S 4$ and 5 which give us the following results. Let $\mathscr{E}$ denote the category whose objects are the short exact sequences of objects in an abelian category

$$
E: 0 \rightarrow K \rightarrow L \rightarrow M \rightarrow 0
$$

where $K$ and $M$ are fixed. The morphisms of $\mathscr{E}$ are all the commutative diagrams:

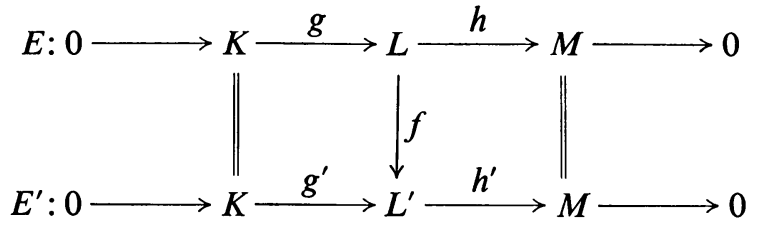

The Grothendieck group, $K_{0}(\mathscr{E}, \boldsymbol{B})$, is shown to be isomorphic to $\operatorname{Ext}^{1}(M, K)$. The Whitehead group, $K_{1}(\mathscr{E}, \boldsymbol{B})$, is computed to be isomorphic to the group

Presented to the Society, August 26, 1970 under the title $K_{0}$ and $K_{1}$ of the category of short sequences; received by the editors January 8, 1971.

AMS 1970 subject classifications. Primary 18F30, 18G15; Secondary 13D15, 18 F25.

Key words and phrases. Algebraic $K$-theory, Baer sum, category of fractions, coherent functor, counit, unit, Grothendieck group, Whitehead group, short exact sequence, selective abelian category, pullback, pushout.

(1) The author gratefully acknowledges the support of the NSF through grant GP-9631 to the Ohio State University.

Copyright (C) 1972, American Mathematical Society 
Hom $(M, K)$. The Grothendieck group of a category of fractions of a category of exact sequences of length $n$ is proven to be $\operatorname{Ext}^{n}(M, K)$.

It is interesting to see just how much of homological algebra can be obtained from algebraic $K$-theory. These computations would lead one to make conjectures about the $K_{-j}$ groups, $j=1,2,3, \ldots$, provided that these could be defined for an abstract category. Gersten has already suggested a cohomology theory for rings which extends the Bass $K_{1}$ and $K_{0}$.

2. Categories of short exact sequences. Let $u: X \rightarrow Y$ and $v: W \rightarrow Y$ be a coterminal pair of morphisms in a category with finite coproducts. The universal property of coproducts then guarantees a unique morphism $\langle u, v\rangle: X \oplus W \rightarrow Y$. Dually, in a category with finite products, we shall denote the unique morphism given by $x: X \rightarrow U$ and $y: X \rightarrow V$ by $\{x, y\}: X \rightarrow U \times V$.

Let $\mathscr{C}$ denote a fixed selective abelian category [4, p. 256]. This means that $\mathscr{C}$ has functions which

(i) assign a unique representative for each subobject,

(ii) assign a unique representative for each quotient object,

(iii) assign to each pair of objects a unique direct sum diagram:

$$
K \underset{\langle 1,0\rangle}{\stackrel{\{1,0\}}{\leftrightarrows}} K \oplus L \underset{\{0,1\}}{\stackrel{\langle 0,1\rangle}{\rightleftarrows}} L
$$

Given a morphism $\{f, g\}: K \rightarrow M \oplus M^{\prime}$, one may denote the selected cokernel by $\langle p,-q\rangle: M \oplus M^{\prime} \rightarrow P$. This means that there is a commutative diagram:

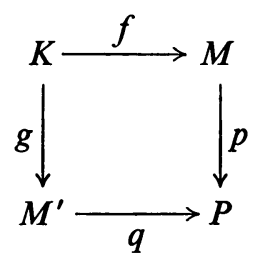

$(P, p, q)$, or simply $P$, is called the selected pushout of $f$ and $g$. Dually, one obtains selected pullbacks.

We shall use the following universal property of pushouts (dually pullbacks) very often: if one has a pushout square $p f=q g$, and if $t f=s g$,

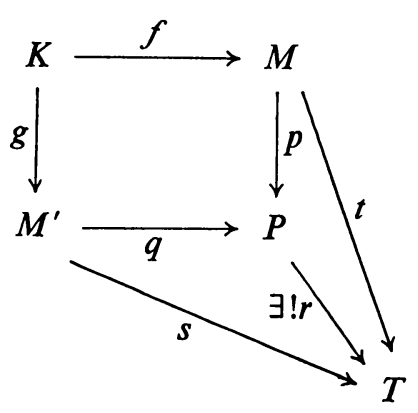


then there is a unique morphism $r: P \rightarrow T$ such that $r q=s$ and $r p=t$. Consider the commutative diagram below:

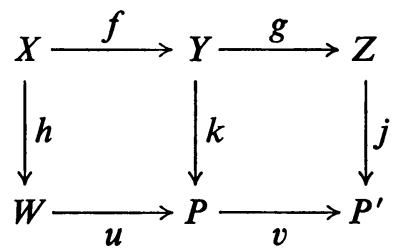

Suppose that $(P, k, u)$ is the selected pushout of $f$ and $h$, and that $\left(P^{\prime}, j, v\right)$ is the selected pushout of $g$ and $k$. Then $\left(P^{\prime}, j, v u\right)$ is a pushout of $g f$ and $h$ [3], but is not necessarily the selected pushout. They differ by an isomorphism.

Since $\mathscr{C}$ is selective, the operation $\oplus$ can be made into a well-defined functor $\oplus: \mathscr{C} \times \mathscr{C} \rightarrow \mathscr{C}$. Mac Lane [5], [6] has noted that this functor is coherently associative and commutative. Let $\tau: \mathscr{C} \times \mathscr{C} \rightarrow \mathscr{C} \times \mathscr{C}$ be the functor defined by $\tau(X, Y)$ $=(Y, X)$ and $\tau(f, g)=(g, f)$. To say that $\oplus$ is associative means that there is a natural isomorphism $a^{\oplus}: \oplus(1 \times \oplus) \rightarrow \oplus(\oplus \times 1): \mathscr{C} \times \mathscr{C} \times \mathscr{C} \rightarrow \mathscr{C}$ such that for any $(X, Y, Z)$ in $\mathscr{C} \times \mathscr{C} \times \mathscr{C}, a^{\oplus}(X, Y, Z)=a^{\oplus}: X \oplus(Y \oplus Z) \stackrel{\cong}{\leftrightarrows}(X \oplus Y) \oplus Z$ is an isomorphism. If for every four objects $A, B, C, D$ of $\mathscr{C}$ the pentagon (2.1) commutes:

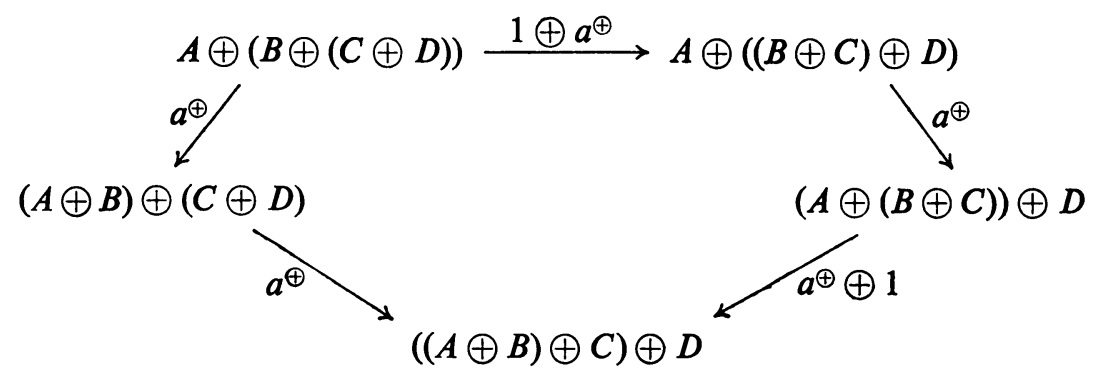

then the associativity isomorphism $a^{\oplus}$ is said to be coherent.

The commutativity isomorphism $c^{\oplus}: \oplus \rightarrow \oplus \tau: \mathscr{C} \times \mathscr{C} \rightarrow \mathscr{C}$, which can be thought of as $c^{\oplus}(X, Y): X \oplus Y \cong Y \oplus X$, is just the morphism $\langle\{0,1\},\{1,0\}\rangle$. Moreover $c^{\oplus}(Y, X) c^{\oplus}(X, Y)=1$.

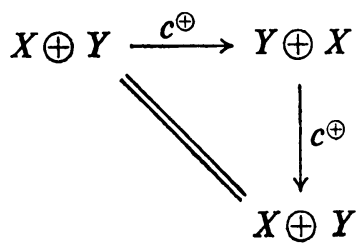


If the diagram (2.3) commutes for all $X, Y$, and $Z$ in $\mathscr{C}$, and if every instance of (2.1) and (2.2) commute, then $a^{\oplus}$ and $c^{\oplus}$ are called jointly coherent.

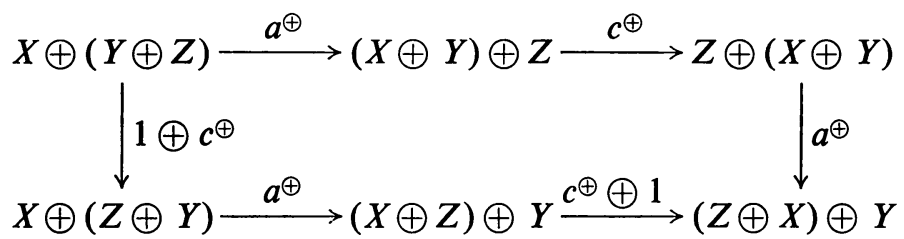

Let us denote by $K^{n}$ the $n$-fold iterated product of $K$ with itself in $\mathscr{C}$. There are several possibilities of bracketing $K^{n}$. Let us fix the letters $k$ and $m$ to denote the codiagonal morphism $k=\langle 1,1\rangle: K^{2} \rightarrow K$ and the diagonal morphism $m$ $=\{1,1\}: M \rightarrow M^{2}$. It is clear that there are morphisms $k(k \oplus 1)$ and $k(1 \oplus k): K^{3}$ $\rightarrow K$ which satisfy

$$
k(1 \oplus k)=k(k \oplus 1) a^{\oplus}: K \oplus(K \oplus K) \rightarrow K .
$$

A similar expression holds for $m$. It is not hard to prove that $k c^{\oplus}=k$ and $c^{\oplus} m=m$. Given a specific $K^{n}$ there is an obvious iteration of $k$ 's summed directly with identity maps which produce a morphism $k^{\star}: K^{n} \rightarrow K$ which is the identity on each component of $K$. Dually there is a morphism $m^{\star}: M \rightarrow M^{t}$.

A morphism $h: K^{n} \rightarrow K^{n^{\prime}}$ is said to be allowable if $k^{\star} h=k^{\star}$, where $k^{\star}$ denotes the appropriate morphism in each instance. Allowable morphisms $j: M^{t} \rightarrow M^{t^{\prime}}$ are those morphisms which satisfy $j m^{\star}=m^{\star}$.

Let us consider the short exact sequence (s.e.s.)

$$
E: 0 \longrightarrow K^{n} \stackrel{g}{\rightarrow} L \stackrel{h}{\rightarrow} M^{t} \longrightarrow 0 .
$$

If one takes the selected pushout of $k^{\star}$ and $g$, one obtains a commutative diagram of s.e.s.'s:

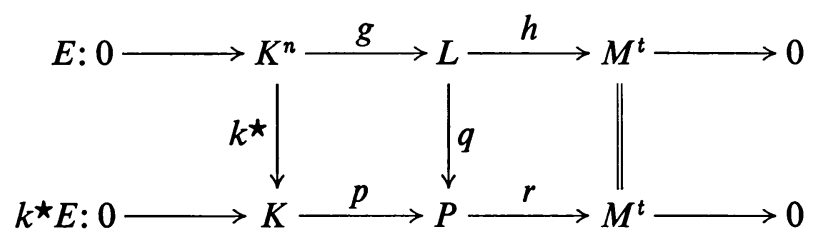

We shall follow Mac Lane's notation [4, p. 65], by calling the newly formed s.e.s. $k^{\star} E$, which is the s.e.s. obtained by pushing out $E$ along $k^{\star}$. Dually, if one pulls $E$ back along $m^{\star}$ by means of the selected pullback, we call the new s.e.s. $E m^{\star}$.

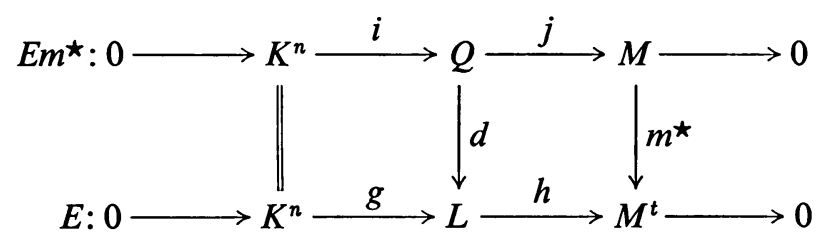


A morphism of s.e.s.'s of $\mathscr{C}$ is a triple $(u, v, w)$ of morphisms giving the usual commutative diagram. Mac Lane [4, p. 66] has noted that $(u, v, w): E^{\prime} \rightarrow E^{\prime \prime}$ can be factored into $\left(u, v^{\prime}, 1\right): E^{\prime} \rightarrow E^{\prime \prime} w$ and $\left(1, v^{\prime \prime}, w\right): E^{\prime \prime} w \rightarrow E^{\prime \prime}$ where the former is a pushout along $u$ of the form of (2.4), and the latter a pullback along $w$ of the form (2.5). (Neither need be the selected pushout or pullback; they may each differ by a congruence of the form $(1, y, 1)$.)

Let $\mathscr{F}$ denote the category whose objects are all s.e.s.'s of the form of $E$ for a fixed $K$, a fixed $M$, for all positive integers $n, t \geqq 1$, and for all possible bracketings of $K^{n}$ and $M^{t}$. The morphisms are all the morphisms of s.e.s.'s $(u, v, w)$ where $u: K^{n} \rightarrow K^{n^{\prime}}$ and $w: M^{t} \rightarrow M^{t^{\prime}}$ are both allowable isomorphisms.

Let $\mathscr{E}$ denote the subcategory of $\mathscr{F}$ for which $n=t=1$ and the morphisms are all of the form $(1, y, 1)$. By the 5-lemma $y$ must be an isomorphism in each instance.

It is clear that the functor $\oplus$ can be extended to act on $\mathscr{F}$ term-by-term, and that $\oplus: \mathscr{F} \times \mathscr{F} \rightarrow \mathscr{F}$ has $a^{\oplus}$ and $c^{\oplus}$ jointly coherent.

Let $(u, v, w): E \rightarrow E^{\prime}$ be a morphism in $\mathscr{F}$. We shall show that this induces a unique morphism $k^{\star} E \rightarrow k^{\star} E^{\prime}$. To see this let us examine the diagram (2.6). Since $u$ is allowable, $k^{\star} u=k^{\star}: K^{n} \rightarrow K$. Therefore, since $q^{\prime} v g=q^{\prime} g^{\prime} u=p^{\prime} k^{\star} u=p^{\prime} k^{\star}$ give two morphisms $K^{n} \rightarrow L \rightarrow P^{\prime}=K^{n} \rightarrow K \rightarrow P^{\prime}$, there is a unique morphism $s: P \rightarrow P^{\prime}$ such that $s p=p^{\prime}$ and $s q=q^{\prime} v$.

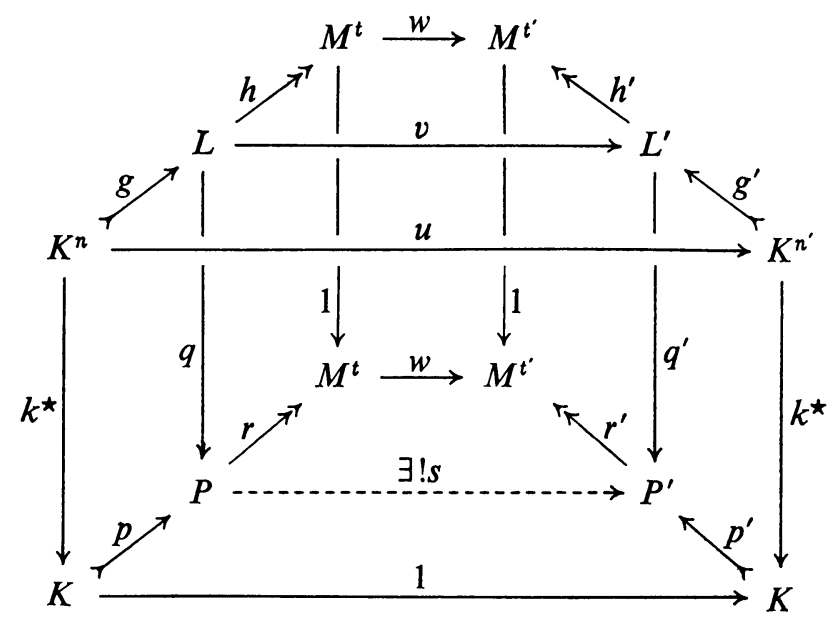

Moreover, since $k^{\star}$ is an epimorphism, it follows from the 5-lemma that $q$ is also an epimorphism. But $w r q=w h=h^{\prime} v=r^{\prime} q^{\prime} v=r^{\prime} s q$, so $w r=r^{\prime} s$, and thus all of (2.6) is commutative.

Let $\nabla: \mathscr{F} \rightarrow \mathscr{F}$ be the covariant functor defined by $\nabla(E)=k^{\star} E$ and $\nabla(u, v, w)$ $=(1, s, w)$. This is well defined. It is easily checked that $\nabla(1,1,1)=(1,1,1)$ and that $\nabla\left(u u^{\prime}, v v^{\prime}, w w^{\prime}\right)=\nabla\left[(u, v, w)\left(u^{\prime}, v^{\prime}, w^{\prime}\right)\right]=\nabla(u, v, w) \nabla\left(u^{\prime}, v^{\prime}, w^{\prime}\right)$. The commutative diagram (2.6) guarantees the existence of a unit, $\eta: \mathbf{1}_{\mathscr{F}} \rightarrow \nabla$, where $\eta$ is a 
natural transformation of functors. One could call $\eta(E)=\left(k^{\star}, q, 1\right)$ and $\eta(u, v, w)$ $=(1, s, w)$ in $(2.6)$.

By duality, there is a covariant functor $\triangle: \mathscr{F} \rightarrow \mathscr{F}$, where $\triangle(E)=E m^{\star}$, and a counit $\varepsilon: \triangle \rightarrow \mathbf{1}_{\mathscr{F}}$.

REMARK. One can "select" pushouts and pullbacks so that $\triangle^{2}=\triangle$ and $\nabla^{2}=\nabla$. In this case $(\nabla, 1, \eta)$ is a triple, and $(\triangle, 1, \varepsilon)$ a cotriple.

\section{The Baer functor and its properties.}

3.1. Definition of the Baer functor. Let $J: \mathscr{E} \rightarrow \mathscr{F}$ denote the inclusion functor. The composite functor

$$
\triangle \nabla \oplus(\boldsymbol{J} \times \boldsymbol{J}): \mathscr{E} \times \mathscr{E} \rightarrow \mathscr{F}
$$

is just a restatement of the usual notion of the Baer sum of two short exact sequences. Moreover, this composite functor can be uniquely factored through $\boldsymbol{J}$. If $E_{1}$ and $E_{2}$ are objects in $\mathscr{E}$, then so is $\triangle \nabla\left(E_{1} \oplus E_{2}\right)$. Similarly,

$$
\triangle \nabla\left((1, y, 1) \oplus\left(1, y^{\prime}, 1\right)\right)=\left(1, y^{\prime \prime}, 1\right)
$$

Therefore, there is a unique functor

$$
\boldsymbol{B}: \mathscr{E} \times \mathscr{E} \rightarrow \mathscr{E}
$$

such that $\boldsymbol{J} \boldsymbol{B}=\triangle \nabla \oplus(\boldsymbol{J} \oplus \boldsymbol{J})$. $\boldsymbol{B}$ is called the Baer functor.

3.2. $B$ is associative. In the following, we shall refer to the arbitrary but fixed s.e.s.'s of $\mathscr{E}$ :

$$
E_{j}: 0 \rightarrow K \rightarrow L_{j} \rightarrow M \rightarrow 0, \quad 1 \leqq j \leqq 4
$$

The composite functor $\boldsymbol{B}\left(\mathbf{1}_{\mathscr{E}} \times \boldsymbol{B}\right): \mathscr{E} \times \mathscr{E} \times \mathscr{E} \rightarrow \mathscr{E}$ can be written as follows (ignoring $J$ ):

$$
\begin{aligned}
\boldsymbol{B}\left(\mathbf{1}_{8} \times \boldsymbol{B}\right)\left(E_{1}, E_{2}, E_{3}\right) & =\boldsymbol{B}\left(E_{1}, \boldsymbol{B}\left(E_{2}, E_{3}\right)\right)=\Delta \nabla \oplus\left(E_{1}, \nabla \Delta \oplus\left(E_{2}, E_{3}\right)\right) \\
& =\Delta \nabla\left(E_{1} \oplus \triangle \nabla\left(E_{2} \oplus E_{3}\right)\right) \\
& =\Delta \nabla\left(\mathbf{1}_{8} \oplus \triangle\right)\left(\mathbf{1}_{8} \oplus \nabla\right)\left(E_{1} \oplus\left(E_{2} \oplus E_{3}\right)\right) .
\end{aligned}
$$

The final form of this expression is expressed diagrammatically by the upper zigzag path of (3.1). The lower zigzag does the same analysis for $\boldsymbol{B}(\boldsymbol{B} \times \mathbf{1})$. Each morphism on these paths denotes selected pullbacks or pushouts given by the unit $\eta$ and counit $\varepsilon$. The morphism $\nabla(1 \oplus \varepsilon)$ could be called $\eta(1 \oplus \varepsilon)$. 


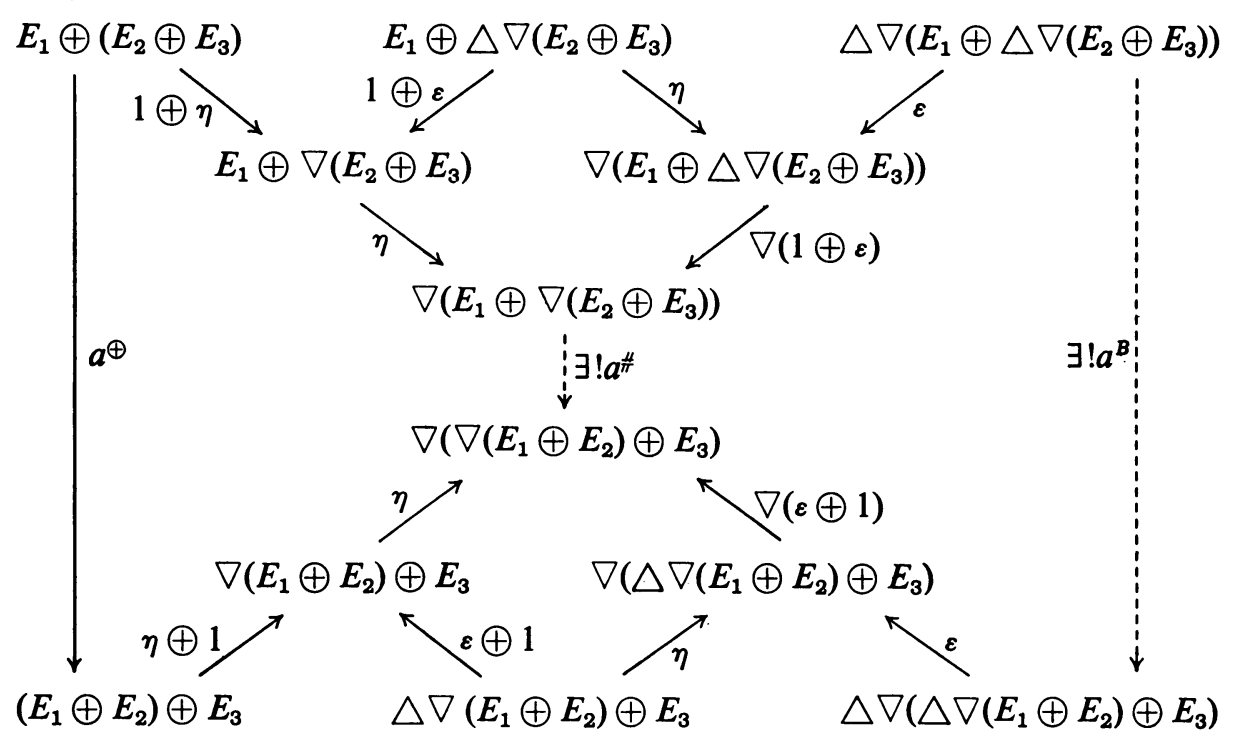

The commutativity of the upper and lower diamond-shaped figures corresponds precisely to the commutativity of diagram (2.6) or to the existence of the unit $\eta$. The morphisms $\nabla(1 \oplus \varepsilon)$ and $\nabla(\varepsilon \oplus 1)$ correspond to the morphisms which are uniquely determined in (2.6), or the action of the functor $\nabla$. These are both pullbacks, but not necessarily the selected ones.

$\nabla\left(E_{1} \oplus \nabla\left(E_{2} \oplus E_{3}\right)\right)$ is a pushout of $E_{1} \oplus\left(E_{2} \oplus E_{3}\right)$ along $k(1 \oplus k)$ (which is our $k^{\star}$ here). By the argument of (2.6) this induces a unique morphism

$$
a^{\#}=\left(1, s, a^{\oplus}\right): \nabla\left(E_{1} \oplus \nabla\left(E_{2} \oplus E_{3}\right)\right) \rightarrow \nabla\left(\nabla\left(E_{1} \oplus E_{2}\right) \oplus E_{3}\right) .
$$

It is easy to verify that $s$ and $a^{\#}$ are isomorphisms. Dually, $\triangle \nabla\left(\triangle \nabla\left(E_{1} \oplus E_{2}\right) \oplus E_{3}\right)$ is a pullback of $\nabla\left(\nabla\left(E_{1} \oplus E_{2}\right) \oplus E_{3}\right)$ along $m(m \oplus 1)=m^{\star}$, so there is a unique morphism $a^{B}=(1, y, 1): \boldsymbol{B}(\mathbf{1} \times \boldsymbol{B})\left(E_{1}, E_{2}, E_{3}\right) \rightarrow \boldsymbol{B}(\boldsymbol{B} \times \mathbf{1})\left(E_{1}, E_{2}, E_{3}\right)$. In fact $a^{\boldsymbol{B}}$ is an isomorphism, with inverse $\left(1, y^{-1}, 1\right)$.

It is well known that the category of morphisms of $\mathscr{C}$ and the category whose objects are the commutative squares in $\mathscr{C}$ are both abelian since $\mathscr{C}$ is abelian [8]. Therefore these categories have pushouts and pullbacks too. The construction of $a^{\#}$ and $a^{B}$ is equivalent to taking a pushout and a pullback in the category of morphisms of $\mathscr{C}$. If $d_{j}: E_{j} \rightarrow E_{j}^{\prime}$ are morphisms in $\mathscr{E}, 1 \leqq j \leqq 3$, then there will be a diagram (3.1)' analogous to (3.1) and morphisms (3.1) $\rightarrow(3.1)^{\prime}$ making everything commute. The commutativity at the $a^{\#}$ and $a^{B}$ level is equivalent to the existence of pushouts and pullbacks in the category of commutative squares.

It follows from all this that

$$
a^{B}\left(E_{1}, E_{2}, E_{3}\right): \boldsymbol{B}(\mathbf{1} \times \boldsymbol{B})\left(E_{1}, E_{2}, E_{3}\right) \rightarrow \boldsymbol{B}(\boldsymbol{B} \times \mathbf{1})\left(E_{1}, E_{2}, E_{3}\right)
$$


can be thought of as a natural transformation of functors

$$
a^{B}: B(\mathbf{1} \times B) \rightarrow B(B \times \mathbf{1})
$$

Since $a^{B}\left(E_{1}, E_{2}, E_{3}\right)$ is an isomorphism in each instance, $a^{B}$ is a natural isomorphism. This is the associativity isomorphism for $\boldsymbol{B}$.

In the same vein, $a^{\#}$ is the associativity isomorphism for the functor $\nabla \oplus: \mathscr{F} \times \mathscr{F}$ $\rightarrow \mathscr{F}$.

3.3. $B$ is coherently associative. Suppose that the pentagon (2.1) is constructed in $\mathscr{F}$ with $E_{1}, E_{2}, E_{3}, E_{4}$ replacing $A, B, C$, and $D$. To show that $a^{B}$ is coherent, it is necessary to construct a commutative pentagon with $\boldsymbol{B}$ replacing $\oplus$.

We shall write the necessary diagram below with the following changes of notation: we shall write $E_{j}$ as $j, E_{j} \oplus E_{k}$ as $j k, \nabla\left(E_{j} \oplus E_{k}\right)$ as $j \nabla k, \boldsymbol{B}\left(E_{j}, E_{k}\right)$ as $j \boldsymbol{B} k$, and 1: $E_{j} \rightarrow E_{j}$ as $e_{j}$. Notice that all vertical morphisms are isomorphisms.

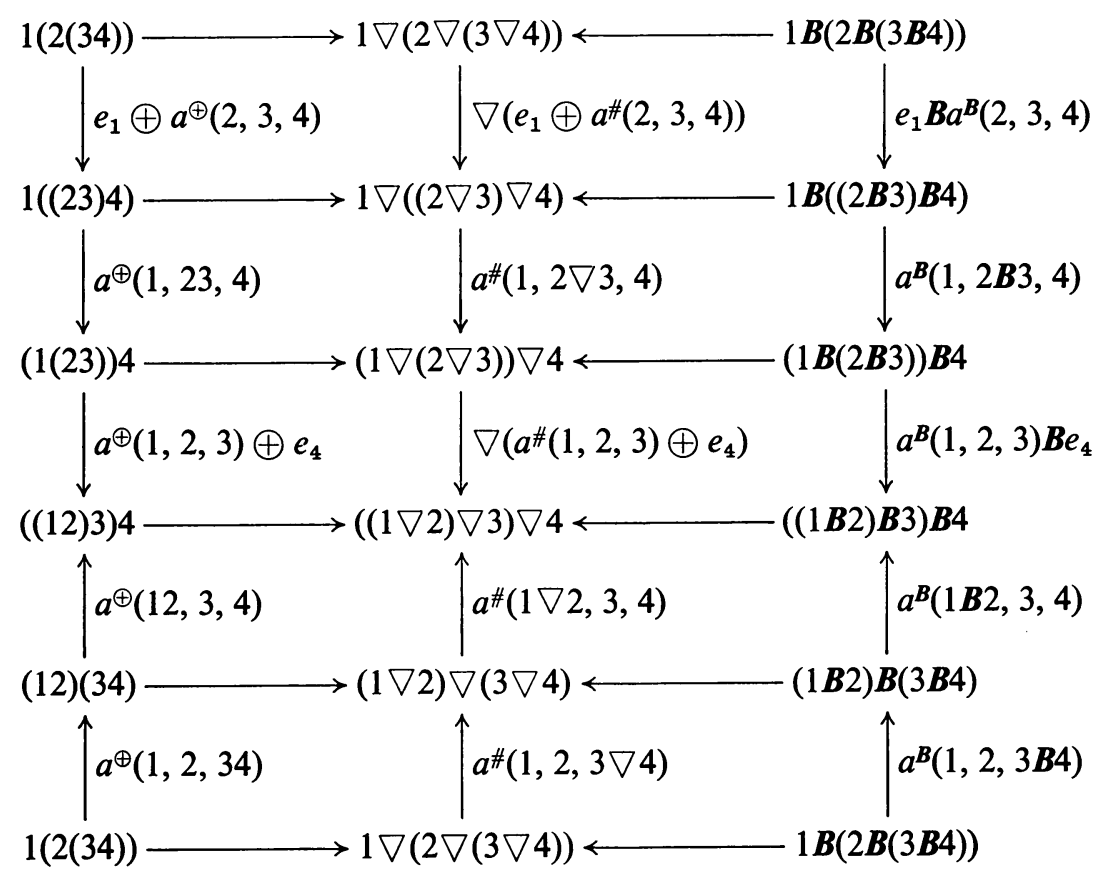

The top left-hand corner square commutes because of the commutativity of the diagram:

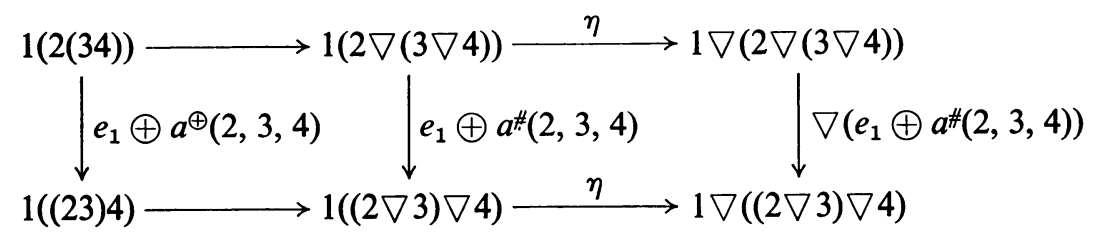


All horizontal morphisms are unique compositions of $\eta$ 's and $\varepsilon$ 's. The second from the top square on the left commutes because the following diagram is commutative:

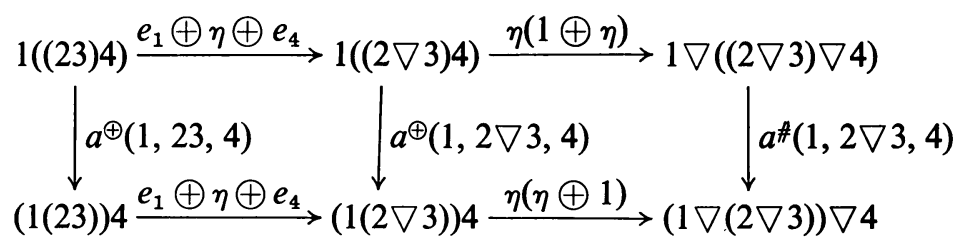

All the other squares are similarly commutative. There are two composite morphisms $1(2(34)) \rightarrow((12) 3) 4$, one from the top and one from the bottom. They are the same by the coherence of $a^{\oplus}$. Therefore they both induce the same morphism $1 \nabla(2 \nabla(3 \nabla 4)) \rightarrow((1 \nabla 2) \nabla 3) \nabla 4$. The diagram gives two obvious candidates for such a morphism; from the uniqueness guaranteed by the universality of pushouts, they must be the same. Similarly, the two morphisms $1 \boldsymbol{B}(2 \boldsymbol{B}(3 \boldsymbol{B} 4)) \rightarrow((1 B 2) B 3) B 4$ must be the same. This is the required pentagon condition. Therefore $a^{B}$. is coherently associative.

3.4. B is commutative. The twisting functor $\tau: \mathscr{C} \times \mathscr{C} \rightarrow \mathscr{C} \times \mathscr{C}$ and the commutativity isomorphism $c^{\oplus}: \oplus \rightarrow \oplus \tau$ can be extended to $\mathscr{E}$ and $\mathscr{F}$ respectively term-by-term, i.e. $c^{\oplus}\left(E_{1}, E_{2}\right)=\left(c^{\oplus}, c^{\oplus}, c^{\oplus}\right): E_{1} \oplus E_{2} \rightarrow E_{2} \oplus E_{1}$. Let us define $c^{B}: B \rightarrow B \tau$ by

$$
c^{B}\left(E_{1}, E_{2}\right)=\triangle \nabla\left(c^{\oplus}, c^{\oplus}, c^{\oplus}\right): B\left(E_{1}, E_{2}\right) \rightarrow \boldsymbol{B}\left(E_{2}, E_{1}\right) .
$$

Since each morphism $c^{\oplus}$ is an isomorphism, so is $c^{\boldsymbol{B}}\left(E_{1}, E_{2}\right)$. Therefore $c^{\boldsymbol{B}}: \boldsymbol{B} \rightarrow \boldsymbol{B} \tau$ is a natural isomorphism. Since $c^{\oplus} c^{\oplus}=1$ in $\mathscr{C}$,

$$
c^{B} c^{B}=\Delta \nabla\left(c^{\oplus}, c^{\oplus}, c^{\oplus}\right) \Delta \nabla\left(c^{\oplus}, c^{\oplus}, c^{\oplus}\right)=\Delta \nabla\left(c^{\oplus} c^{\oplus}, c^{\oplus} c^{\oplus}, c^{\oplus} c^{\oplus}\right)=1 .
$$

Therefore $c^{\boldsymbol{B}}$ is a commutativity isomorphism.

It is worthwhile looking at $c^{B}$ in the following manner:

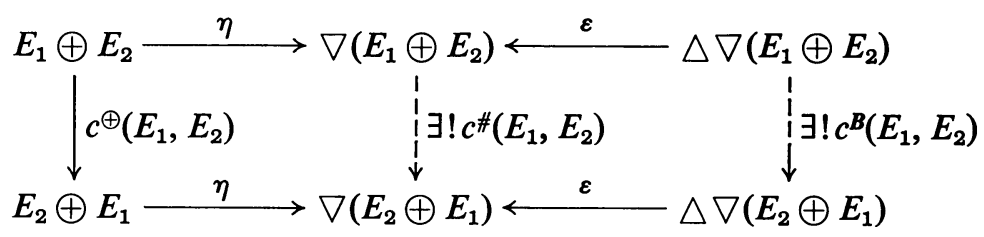

Since $\nabla\left(E_{1} \oplus E_{2}\right)$ is the selected pushout of $E_{1} \oplus E_{2}$ along $k$, there is a unique morphism $c^{\#}\left(E_{1}, E_{2}\right)$ such that $c^{\#} \eta=\eta c^{\oplus}$. Since $\eta$ is a unit, $c^{\#}=\nabla\left(c^{\oplus}\left(E_{1}, E_{2}\right)\right)$ $=\nabla\left(c^{\oplus}, c^{\oplus}, c^{\oplus}\right)$. Dually there is a unique $c^{B}$ making the second square commute, and $c^{B}=\triangle\left(c^{\#}\right)$.

3.5. $\boldsymbol{B}$ is coherently commutative and associative. It suffices to produce a commutative diagram analogous to (2.3). Let us select $E_{1}, E_{2}$, and $E_{3}$ and use the notation of $\S 3.3$. There is a natural choice of a large diagram which represents the 
diagram (2.3) for $a^{\oplus}$ and $c^{\oplus}$ in its first column, and the corresponding situation for $a^{B}$ and $c^{B}$ in the third column. All vertical morphisms are isomorphisms. The diagram is commutative by the usual argument.

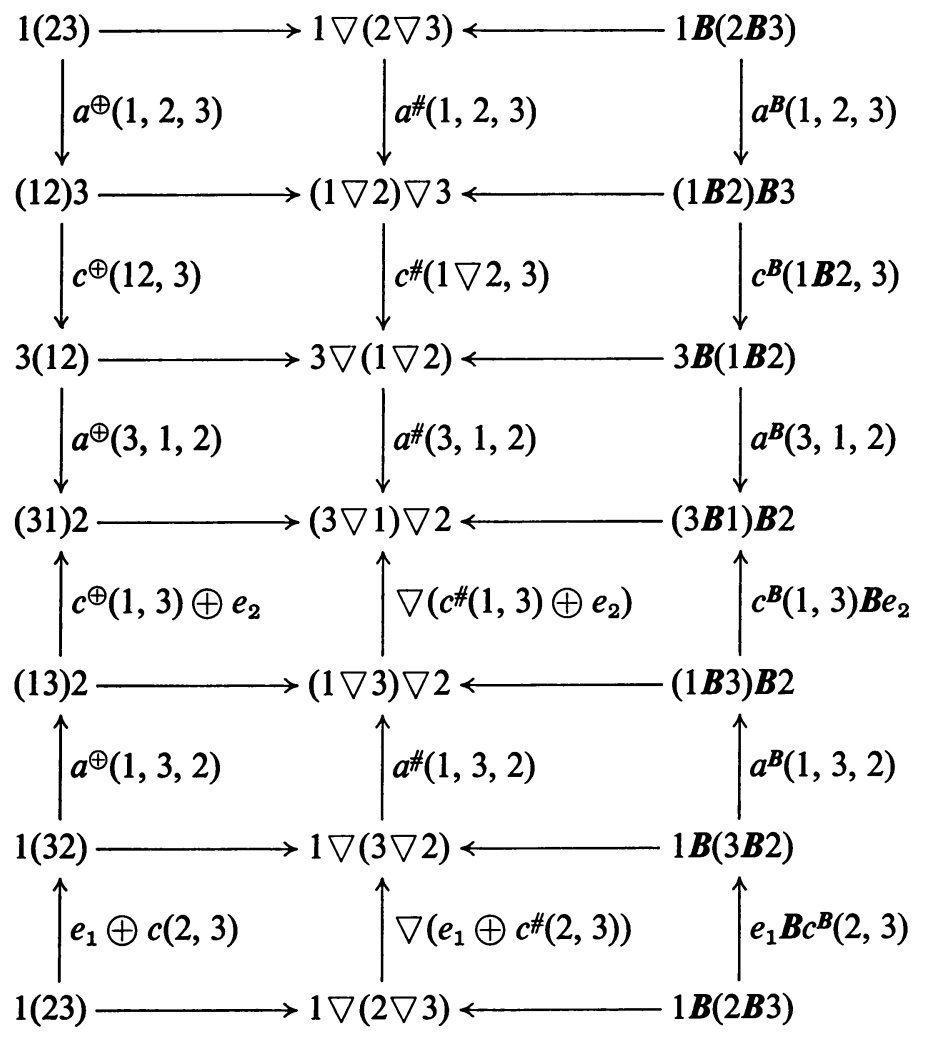

The isomorphism $E_{1} \oplus\left(E_{2} \oplus E_{3}\right) \rightarrow\left(E_{3} \oplus E_{1}\right) \oplus E_{2}$ induces a unique isomorphism $E_{1} B\left(E_{2} B E_{3}\right) \rightarrow\left(E_{3} B E_{1}\right) B E_{2}$. The two candidates in the last column must therefore coincide so $a^{B}$ and $c^{B}$ are jointly coherent.

REMARKS. 1. Let us fix as our ground object the split s.e.s. $S$

$$
S: 0 \longrightarrow K \stackrel{\{1,0\}}{\longrightarrow} K \oplus M \stackrel{\langle 0,1\rangle}{\longrightarrow} M \longrightarrow 0 .
$$

Mac Lane [5] discusses left identity isomorphisms which would require isomorphisms $e: B(S, E) \rightarrow E$ for each $E$. It is not clear that this would be coherent, but fortunately we do not require these identity morphisms here.

2. This proof contains the fact that the functor $\#=\nabla \oplus: \mathscr{F} \times \mathscr{F} \rightarrow \mathscr{F}$ has jointly coherent commutativity and associativity isomorphisms $c^{\#}$ and $a^{\#}$.

3. If $\mathscr{F}_{i}$ is the category whose objects are the exact sequences

$$
0 \rightarrow K^{n} \rightarrow V_{1} \rightarrow \cdots \rightarrow V_{1} \rightarrow M^{t} \rightarrow 0
$$


and whose morphisms $\left(u, v_{1}, \ldots, v_{i}, w\right)$ have $u$ and $w$ allowable, and if $\mathscr{E}_{i}$ is the subcategory with $n=t=1$ and morphisms $\left(1, v_{1}, \ldots, v_{i}, 1\right)$, then the whole procedure works just as well for these categories too. That is, $\mathscr{E}_{i}$ has a Baer functor $\boldsymbol{B}_{i}: \mathscr{E}_{i} \times \mathscr{E}_{i} \rightarrow \mathscr{E}_{i}$ which is a coherently associative and commutative product. $\mathscr{E}$ and $\boldsymbol{B}$ above could be renamed $\mathscr{E}_{1}$ and $\boldsymbol{B}_{1}$.

This can be all summarized as follows:

THEOREM 1. $\boldsymbol{B}_{i}$ is a coherently associative and commutative product on the category $\mathscr{E}_{i}, i=1,2, \ldots$

4. Grothendieck group computations. Since $B$ is coherently associative and commutative we are now able to proceed to compute the Grothendieck group $K_{0}(\mathscr{E}, \boldsymbol{B})$ of $\mathscr{E}$ according to the method of Bass [1].

Since every pair of s.e.s.'s of $\mathscr{E}$ either possess an isomorphism between them or have no morphism at all from one to the other, one can easily form equivalence classes of isomorphic objects. It is well known [4], [10] that under Baer sum these classes form the abelian group $\operatorname{Ext}^{1}(M, K)$. Thus we have proven

THEOREM 2. $K_{0}(\mathscr{E}, B) \cong \operatorname{Ext}^{1}(M, K)$.

Furthermore, $K_{0}(\mathscr{F}, \triangle \nabla \oplus)$ is also equal to $\operatorname{Ext}^{1}(M, K)$. To see this, one need only note that the zero class is represented by the split s.e.s. $S$. If one applies the appropriate functor one finds that for an arbitrary s.e.s. $E$, the isomorphism classes $[E]+[S]=\left[E^{\prime}\right]$ for $E^{\prime}=\triangle \nabla(E \oplus S)$. But $[S]=0$, so $[E]=\left[E^{\prime}\right]$. But $E^{\prime}$ is a s.e.s. in $\mathscr{E}$ so the computation is the same as above.

Let $\Sigma$ denote the family of all morphisms of $\mathscr{E}_{i}$, and let $\mathscr{E}_{i}(\Sigma)$ denote the category of fractions [2]. The latter category is a groupoid, so every morphism is invertible. Moreover, $\boldsymbol{B}_{i}$ can be uniquely extended to be a functor on $\mathscr{E}_{i}(\Sigma)$. (If $f_{1} f_{2}^{-1} f_{3}$ and $f_{4}^{-1} f_{5} f_{6}^{-1}$ are two morphisms of $\mathscr{E}_{i}(\Sigma)$-where the inverse simply denotes a morphism going the wrong way-then let us set

$$
\left.\boldsymbol{B}\left(f_{1} f_{2}^{-1} f_{3}, f_{4}^{-1} f_{5} f_{6}^{-1}\right)=\boldsymbol{B}\left(f_{1}, 1\right) \boldsymbol{B}\left(f_{2}, 1\right)^{-1} \boldsymbol{B}\left(f_{3}, 1\right) \boldsymbol{B}\left(1, f_{4}\right)^{-1} \boldsymbol{B}\left(1, f_{5}\right) \boldsymbol{B}\left(1, f_{6}\right)^{-1} .\right)
$$

The usual argument proves that

$$
K_{0}\left(\mathscr{E}_{i}(\Sigma), \boldsymbol{B}_{i}\right)=\operatorname{Ext}^{i}(M, K) .
$$

5. The Whitehead group of $\mathscr{E}$. It has been proven in [8] that to each automorphism of $E$ in $\mathscr{E}$

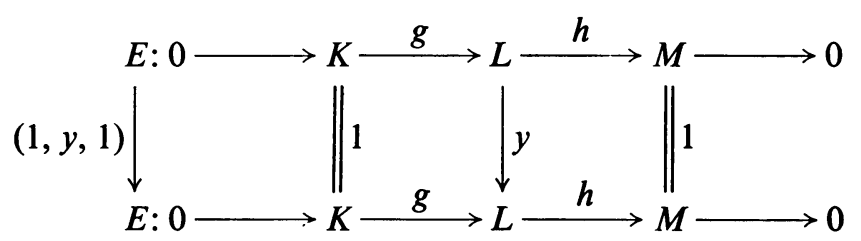

there is a unique morphism $\alpha: M \rightarrow K$ such that $y=1+g \alpha h$. Let us denote this automorphism by $(E, \alpha): E \rightarrow E$. 
$(E, \alpha)$ will be called isomorphic to $\left(E^{\prime}, \alpha^{\prime}\right)$ if there is a morphism $(1, f, 1): E \rightarrow E^{\prime}$ (see $\S 1$ ) such that the diagram involving two copies of $E$ and $E^{\prime}$ commutes. This gives the three equalities:

$$
f(1+g \alpha h)=\left(1+g^{\prime} \alpha^{\prime} h^{\prime}\right) f ; \quad f g=g^{\prime} ; \quad \text { and } \quad h^{\prime} f=h .
$$

The first equality gives $f+f g \alpha h=f+g^{\prime} \alpha^{\prime} h^{\prime} f$, and hence $f g \alpha h=g^{\prime} \alpha^{\prime} h^{\prime} f$, or $g^{\prime} \alpha h$ $=g^{\prime} \alpha^{\prime} h$. But since $h$ is an epimorphism and $g^{\prime}$ is a monomorphism we must have $\alpha=\alpha^{\prime}$. Thus we have proven half of the following result:

LEMmA 1. $(E, \alpha) \cong\left(E^{\prime}, \alpha^{\prime}\right)$ if and only if $E \cong E^{\prime}$ and $\alpha=\alpha^{\prime}$.

Certainly if we are given an isomorphism from $E$ to $E^{\prime}$, and are told that $\alpha=\alpha^{\prime}$, then this will induce an isomorphism between $(E, \alpha)$ and $\left(E^{\prime}, \alpha^{\prime}\right)$.

Take two s.e.s.'s $E_{1}$ and $E_{2}$ as in (3.2), and let $\alpha_{1}$ and $\alpha_{2}$ be morphisms from $M$ to $K$. Since $B$ is a functor, let us compute

$$
\boldsymbol{B}\left(\left(E_{1}, \alpha_{1}\right),\left(E_{2}, \alpha_{2}\right)\right)=\Delta \nabla\left(\left(E_{1}, \alpha_{1}\right) \oplus\left(E_{2}, \alpha_{2}\right)\right) .
$$

It is a straightforward computation to prove that

$$
\nabla\left(\left(E_{1}, \alpha_{1}\right) \oplus\left(E_{2}, \alpha_{2}\right)\right)=\left(\nabla\left(E_{1} \oplus E_{2}\right),\left\langle\alpha_{1}, \alpha_{2}\right\rangle\right)
$$

where $\left\langle\alpha_{1}, \alpha_{2}\right\rangle: M \oplus M \rightarrow K$ is the morphism with components $\alpha_{1}$ and $\alpha_{2}$. It can be verified that after applying $\triangle$, the induced automorphism is given as follows:

LeMmA 2. $B\left(\left(E_{1}, \alpha_{1}\right),\left(E_{2}, \alpha_{2}\right)\right)=\left(B\left(E_{1}, E_{2}\right), \alpha_{1}+\alpha_{2}\right)$.

A short exact sequence $E$ is said to split if there are morphisms $s: M \rightarrow L$ and $t: L \rightarrow K$ such that $h s=1, t g=1$, and $s h+g t=1$. If $E$ splits, there is a commutative diagram

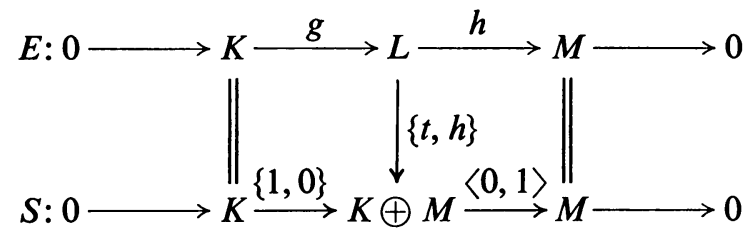

That is, each split s.e.s. is isomorphic to $S$. Given any s.e.s. $E$ there is always a s.e.s. $E^{*}$ such that $B\left(E, E^{*}\right)$ splits, because $\operatorname{Ext}^{1}(M, K)$ is a group. Therefore $\left(B\left(E, E^{*}\right), \alpha\right) \cong(S, \alpha)$ for every $\alpha: M \rightarrow K$.

Another operation which is often associated with automorphisms is composition.

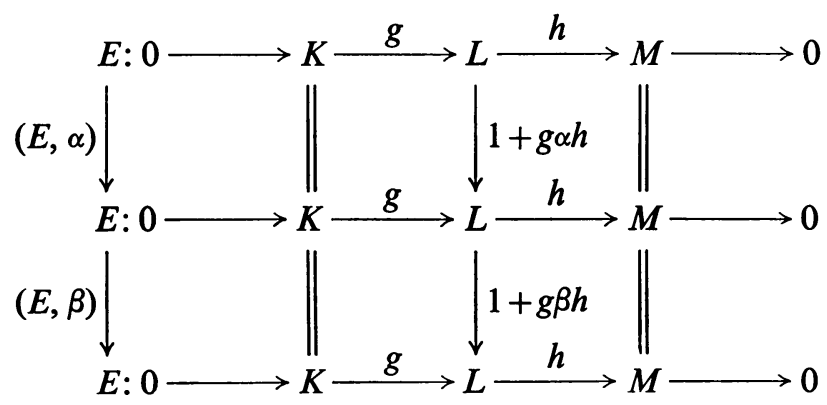


Since $h g=0$ it is clear that $(1+g \beta h)(1+g \alpha h)=1+g(\beta+\alpha) h$. It follows that if one denotes composition by "。”, then

$$
(E, \beta) \circ(E, \alpha)=(E, \beta+\alpha) \text {. }
$$

The Whitehead group of $\mathscr{E}$ has been defined [1] as follows: let $|\Sigma \mathscr{E}|$ denote all the automorphisms of $\mathscr{E}$ and let [ ]: $|\Sigma \mathscr{E}| \rightarrow K_{1}(\mathscr{E}, \boldsymbol{B})$ be a map which is universal for maps into abelian groups which satisfy:

Ka. If $(E, \alpha) \cong\left(E^{\prime}, \alpha^{\prime}\right)$ then $[E, \alpha]=\left[E^{\prime}, \alpha^{\prime}\right]$.

$\mathrm{Kb} .\left[\boldsymbol{B}\left(\left(E_{1}, \alpha_{1}\right),\left(E_{2}, \alpha_{2}\right)\right)\right]=\left[E_{1}, \alpha_{1}\right]+\left[E_{2}, \alpha_{2}\right]$.

Kc. $[(E, \beta) \circ(E, \alpha)]=[E, \beta]+[E, \alpha]$.

Certainly in view of the above remarks these rules can be restated here as follows:

Ka. If $(E, \alpha) \cong\left(E^{\prime}, \alpha\right)$, then $[E, \alpha]=\left[E^{\prime}, \alpha\right]$.

$\mathrm{Kb} .\left[\boldsymbol{B}\left(E_{1}, E_{2}\right), \alpha_{1}+\alpha_{2}\right]=\left[E_{1}, \alpha_{1}\right]+\left[E_{2}, \alpha_{2}\right]$.

Kc. $[E, \beta+\alpha]=[E, \beta]+[E, \alpha]$.

It follows from $\mathrm{Kc}$ that $[E, 0]=[E, 0]+[E, 0]$. Therefore, $[E, 0]=0$ for all $E$. If $S$ is the split s.e.s. given above, then given $E$ and $E^{*}$ such that $B\left(E, E^{*}\right) \cong S$, it follows from $\mathrm{Kb}$ that for any $(E, \alpha)$

$$
\left[\boldsymbol{B}\left(E, E^{*}\right), \alpha\right]=\left[\boldsymbol{B}\left(E, E^{*}\right), \alpha+0\right]=[E, \alpha]+\left[E^{*}, 0\right]=[\widehat{E}, \alpha] .
$$

By Ka, $[S, \alpha]=\left[B\left(E, E^{*}\right), \alpha\right]=[E, \alpha]$.

Proposition $1.6(\mathrm{~b})[1, \mathrm{p} .349]$ proves that $[E, \alpha]=[E, \beta]$ if and only if there are isomorphisms $\left(E_{1}, \gamma\right),\left(E_{2}, \delta_{0}\right),\left(E_{2}, \delta_{1}\right),\left(E_{3}, \varepsilon_{0}\right)$, and $\left(E_{3}, \varepsilon_{1}\right)$ such that the iterated Baer sums

$$
(E, \alpha) \boldsymbol{B}\left(E_{1}, \gamma\right) \boldsymbol{B}\left(E_{2}, \delta_{0}\right) \boldsymbol{B}\left(E_{2}, \delta_{1}\right) \boldsymbol{B}\left(\left(E_{3}, \varepsilon_{0}\right) \circ\left(E_{3}, \varepsilon_{1}\right)\right)
$$

and

$$
(E, \beta) \boldsymbol{B}\left(E_{1}, \gamma\right) \boldsymbol{B}\left(\left(E_{2}, \delta_{0}\right) \circ\left(E_{2}, \delta_{1}\right)\right) \boldsymbol{B}\left(E_{3}, \varepsilon_{0}\right) \boldsymbol{B}\left(E_{3}, \varepsilon_{1}\right)
$$

are isomorphic. If $E=E_{1}=E_{2}=E_{3}=S$, then this would say

$$
\left(S, \alpha+\gamma+\delta_{0}+\delta_{1}+\varepsilon_{0}+\varepsilon_{1}\right) \cong\left(S, \beta+\gamma+\delta_{0}+\delta_{1}+\varepsilon_{0}+\varepsilon_{1}\right) .
$$

By Lemma $1, \alpha=\beta$. In particular, $[S, \alpha]=0=[S, 0]$ if and only if $\alpha=0$.

Let $\mathscr{E}^{\prime}$ be the full subcategory of $\mathscr{E}$ with one object $S$, and let $\boldsymbol{F}: \mathscr{E}^{\prime} \rightarrow \mathscr{E}$ denote the inclusion functor. The functor $\boldsymbol{B}$ still works in $\mathscr{E}^{\prime}$, and $\boldsymbol{F}$ is cofinal, product preserving, and $E$-surjective. By Proposition $2.5[1$, p. 356] there is an exact sequence

$$
K_{1}(F) \rightarrow K_{1}\left(\mathscr{E}^{\prime}\right) \rightarrow K_{1}(\mathscr{E}) .
$$

In addition $K_{1}(\boldsymbol{F})$ is generated by objects $(S, \alpha)$ such that $\boldsymbol{F}((S, \alpha))$ is an identity automorphism. $(S, 0)$ is the only such element, and it has been proven that $[S, 0]$ 
$=0$, so $K_{1}(\boldsymbol{F})=0$. Therefore $K_{1}\left(\mathscr{E}^{\prime}\right)$ is mapped monomorphically into $K_{1}(\mathscr{E})$. Thus we have proven the following:

Lemma 3. $[E, \alpha]=0$ in $K_{1}(\mathscr{E})$ if and only if $\alpha=0$.

We can now state our main theorem of this section.

THEOREM 3. $K_{1}(\mathscr{E}) \cong \operatorname{Hom}(M, K)$.

Proof. Since $[S, \alpha]=[E, \alpha]$ for all $E$, it suffices to look only at the elements $[S, \alpha]$, which we can denote simply by $[\alpha]$. Certainly $[\alpha+\beta]=[\alpha]+[\beta]$. If $\sum n_{i}\left[\alpha_{i}\right]=0$ then by Lemma 3 it follows that $\sum n_{i} \alpha_{i}=0$. Thus there can be no relation among the symbols $[\alpha]$ that does not already exist among the $\alpha$ 's of $\operatorname{Hom}(M, K)$.

In a following paper [9], we shall investigate the six term exact sequence involving these Whitehead and Grothendieck group computations, and compare them with the usual Hom-Ext sequence.

\section{REFERENCES}

1. H. Bass, Algebraic K-theory, Benjamin, New York, 1968. MR 40 \#2736.

2. P. Gabriel and M. Zisman, Calculus of fractions and homotopy theory, Ergebnisse der Mathematik und ihrer Grenzgebiete, Band 35, Springer-Verlag, New York, 1967. MR 35 \#1019.

3. P. Hilton, Correspondences and exact squares, Proc. Conference Categorical Algebra (La Jolla, Calif., 1965), Springer, New York, 1966, pp. 254-271. MR 34 \#4326.

4. S. Mac Lane, Homology, Die Grundlehren der math. Wissenschaften, Band 114, Academic Press, New York; Springer-Verlag, Berlin, 1963. MR 28 \#122.

5. - Natural associativity and commutativity, Rice Univ. Studies 49 (1963), no. 4, 28-46. MR 30 \#1160.

6. - Categorical algebra, Bull. Amer. Math. Soc. 71 (1965), 40-106. MR 30 \#2053.

7. I. S. Pressman, Functors whose domain is a category of morphisms, Acta Math. 118 (1967), 223-249. MR 35 \#4279.

8. - Endomorphisms of exact sequences, Bull. Amer. Math. Soc. 77 (1971), 239-242.

9. - Whitehead groups of categories of short exact sequences (to appear).

10. N. Yoneda, On Ext aild exact sequences, J. Fac. Sci. Univ. Tokyo Sect. I 8 (1960), 507-576. MR 37 \#1445.

Ohio State University, Columbus, Ohio 43210 